\title{
Evaluation of Sleep Problems or Disorders Using Sleep Questionnaires
}

\author{
Nayeong Kong, Jinhui Choi, and Wan Seok Seo \\ Department of Psychiatry, Yeungnam University College of Medicine, Yeungnam University Hospital, Daegu, Korea
}

\begin{abstract}
Many people have various sleep problems or disorders. It is important to administer the appropriate sleep questionnaire for early diagnosis of sleep problems or disorders. Although sleep questionnaires are less accurate than polysomnography, they are inexpensive and easy to apply, making them useful for patients in primary care settings. They are also useful when following up on a treatment, when conducting a screening test for a specific population, and when using them as research tools. Some sleep questionnaires have recently been developed as a mobile app, making them easier to use. In this paper, we will review some sleep questionnaires used by physicians to diagnose sleep problems or disorders.
\end{abstract}

Key Words: Sleep; Sleep Wake Disorders; Surveys and Questionnaires; Mass Screening

Received: November 8, 2019 Revised: December 12, 2019 Accepted: December 13, 2019

Corresponding author: Wan Seok Seo, MD, PhD, Department of Psychiatry, Yeungnam University College of Medicine, Yeungnam University Hospital, 170 Hyunchung-ro, Nam-gu, Daegu 42415, Korea.

Tel: 82-53-620-3344, Fax: 82-53-657-3921, E-mail: sws3901@ynu.ac.kr

(a) This is an Open Access article distributed under the terms of the Creative Commons Attribution Non-Commercial License (https://creativecommons.org/licenses/bync/4.0) which permits unrestricted non-commercial use, distribution, and reproduction in any medium, provided the original work is properly cited.

\section{INTRODUCTION}

Sleep is an important physiological process of cognitive and physical functioning in humans. Sleep deprivation and sleep disorders have negative effects on medical, physiological, behavioral, and academic functions in humans. Many modern people describe negative effects of chronic sleep and wakefulness difficulties on work, academics, and driving activity as well as physical and emotional health [1,2]. Lee et al. [3] reported that the loss in productivity owing to sleep problems costs approximately 10 billion dollars a year in Korea.

Sleep disorders were classified into seven major categories in the third edition of the International Classification of Sleep Disorders (ICSD) released in 2014 [4]. Most sleep-related complaints in clinical situations are as follows: insomnia, excessive sleepiness, sleep behavior, or irregular sleep-wake schedule [5].

Early diagnosis and intervention of various sleep problems or disorders are important. The gold standard for evaluating sleep disorders is polysomnogram (PSG). PSG is a complete lab-based monitoring tool that can accurately assess various sleep disorders. It can be used to diagnose sleep disorders by analyzing sleep stages, breathing patterns, and movements during specific sleep stages using records from many variables (e.g., electroencephalography, electromyography, electrooculography) [6].

Meanwhile, the first step in diagnosing sleep disorders is history taking. History taking provides information about the patient's chief complaints, onset, and precipitating factors in their sleep problems, as well as sleep hygiene, substance use such as alcohol and caffeine, and night time lifestyle. However, this process can vary greatly depending on the physician's knowledge and experience with regards to sleep physiology and sleep disorders. Additional assessment tools should be used to complement history taking when diagnosing sleep disorders.

The primary tools for evaluating a person's sleep patterns or sleep problems are sleep questionnaires and sleep diaries. A sleep diary is a simple tool used to track a person's sleep patterns, usually completed within 1-2 minutes. Sleep diary entries include: time to go to sleep; time to wake up; how long taken to fall asleep; sleep duration; number, times and durations of awakening; presence of a nap during the day; exercise; medication, caffeine or alcohol use; etc. [5]. Meanwhile, a sleep questionnaire is a commonly used tool for the first diagnostic test in a primary care setting and provides a general measure of subjective quality of sleep. Sleep questionnaires have several advantages over sleep diaries. They have a short evaluation time, need no professional assistance, and no specific devices are required [7]. 
This paper reviews the purpose and utilization of sleep questionnaires, which are widely used in clinical settings, for helping general physician or sleep non-specialist to help diagnose patients.

\section{GENERAL SLEEP ASSESSMENT}

\section{Sleep-Wake Activity Inventory}

Sleep-Wake Activity Inventory (SWAI) was developed by Rosenthal et al. [8] in 1993, and consists of six subscales evaluating sleepiness, nocturnal sleep, ability to relax, energy level, social desirability, and psychic distress over the previous 7 days [8]. It can evaluate general aspects of sleep and awakening in the patients. SWAI consists of a total of 59 items. Each item is composed of semi-continuous Likert with 1-9 points ( $1=$ always, $9=$ never). SWAI-Excessive daytime sleepiness (EDS) is used for evaluating excessive daytime sleep; a score below 40 indicates excessive sleepiness, a score 50 or above is normal, and a score between 40 and 50 means possible sleepiness. SWAI has well been associated with multiple sleep latency test (MSLT), which are considered the gold standard tests of sleepiness. It is also known to be useful in determining the treatment effect of obstructive sleep apnea (OSA) [9].

\section{Sleep Disorders Questionnaire}

Sleep Disorders Questionnaire (SDQ) is a screening tool for evaluation of various sleep disturbances and sleep habits over the previous six months. SDQ was developed by Douglas et al. [10]. It consist of a long questionnaire including 175 items for assessing common sleep disorders such as sleep apnea, narcolepsy, psychiatric sleep disorder, and periodic limb movement disorder over previous six months. Each item can be scored from 1 (never) to 5 (always). SDQ was initially designed to help general practitioners diagnose sleep disorders. The developers have also created a smaller, 45-item version of the scale to assess four common sleep disorders.

\section{Dysfunctional Beliefs and Attitudes about Sleep Scale}

The original Dysfunctional Beliefs and Attitudes about Sleep Scale (DBAS) consists of a 30-item self-report questionnaire to evaluate sleep-disruptive cognition. Morin et al. [11] also created a 16-item based brief version of DBAS in 2007. It measures the person's sleep related cognitions (e.g., faulty beliefs and appraisals, unrealistic expectations, perceptual and attention bias) using 100$\mathrm{mm}$ visual analog scales. A high score indicates a person having extensive false beliefs about sleep. This scale is useful for evaluating an individual's sleep-related beliefs, attitudes to insomnia, and for evaluating treatment outcomes according to cognitive changes in the patient. A Korean version of DBAS was validated by Yu et al. [12] in 2009.

\section{INSOMNIA DISORDERS}

\section{Pittsburgh Sleep Quality Index}

Pittsburgh Sleep Quality Index (PSQI) was developed by Buysse et al. [13] and is the most widely used tool to evaluate sleep quality and sleep disturbance. It consists of 7 components (subjective sleep quality, sleep latency, sleep duration, habitual sleep efficiency, sleep disturbance, use of sleeping medication, and daytime dysfunction) with a total of 24 items and takes about 5-10 minutes to complete. The 24 items consist of 19 items directly assessed by the patients and 5 items evaluated by the bed partners. Each item is rated from 0 to 3 points, with a total score ranging from 0 to 21 points. If the score is 5 or more, individuals are determined to be bad sleepers; if it is less than 5 , they are evaluated as good sleepers. PSQI has been used in the diagnosis of various sleep and insomnia disorders. PSQI was validated as a Korean version [14].

\section{Insomnia Severity Index}

Insomnia Severity Index (ISI) was developed by Morin et al. to evaluate insomnia according to the diagnostic criteria of Diagnostic and Statistical Manual of Mental Disorders, 4th edition and ICSD [15]. ISI is consists of 7 self-reported items (severity of sleep onset, severity of sleep maintenance, severity of early morning awakening problems, sleep dissatisfaction, interference of sleep difficulties with daytime functioning, noticeability of sleep problems by others, distress caused by the sleep difficulties) that assess the nature, severity, and impact of insomnia over the previous month. ISI is rated on a five-point Likert scale from 0 to 4 points per item with a total score of $0-28$ points. A score below 7 indicates no insomnia, that between 8 and 14 indicates insomnia below threshold, that between 15 and 21 means moderate insomnia, and that over 22 indicates severe insomnia. It was translated into a Korean version [16]. This questionnaire can be applied to individuals of all ages and is useful for psychiatric patients who have insomnia symptoms.

\section{HYPERSOMNOLENCE DISORDERS}

\section{Epworth Sleepiness Scale}

Epworth Sleepiness Scale (ESS) is a self-rating scale of sleepiness in eight situations developed by Johns [17] and sensitively reflects a person's sleepiness. In each situation, the degree of sleepiness is assessed from 0 to 3 points, and the total score ranges from 0 to 24 points. A total score of 10 or above indicates a serious case of EDS. It was translated into a Korean version and is widely used in Korean hospitals [18]. Although correlation studies with MSLT are diverse, there are questions about whether ESS can be used as a measure of drowsiness. Because of the Korean unique lifestyle, modifications are necessary to improve the measurement accuracy of the ESS. Most of the respondents were either confused or hesitant to answer certain questions that refer to situations involving sitting and driving. Very recently, Jung et al. [19] developed and validated a modified Korean ESS that reflects the Korean lifestyle.

\section{Stanford Sleepiness Scale}

Stanford Sleepiness Scale (SSS) was developed by Hoddes et al. [20] and is useful for evaluating acute sleepiness through the day. 
It evaluates the degree of sleepiness and alertness on a scale of 1 to 7 ( 1 =feeling active, vital, alert, and wide awake, $7=$ almost in reverie and cannot stay awake. Sleep onset is imminent). SSS is very easy to apply, and it can be used several times a day.

\section{BREATHING-RELATED SLEEP DISORDERS}

\section{Berlin Questionnaire}

Berlin Questionnaire (BQ) was developed by respiratory and primary care physicians through consensus to evaluate OSA, and it was validated in 1996. BQ consists of 10 questions in three categories related to snoring, daytime sleepiness, and obesity or hypertension [21,22]. If positive more than two of the three categories fall into the OSA high-risk group. Category 1 consists of five questions related to snoring and apnea during sleep. It is considered positive when answering yes to 2 or more questions. Category 2 consists of four questions related to sleepiness and tiredness. It is considered positive when answering yes to 2 or more questions. Category 3 is considered positive when the individual has high blood pressure or a BMI $>30 \mathrm{~kg} / \mathrm{m}^{2}$. BQ was translated into a Korean version [23].

\section{STOP questionnaire}

STOP questionnaire was developed by the University of Toronto for screening OSA in 2008 [24]. The eight items including snoring, tiredness during daytime, observed apnea, and high blood pressure are rated as yes or no. Combined with BMI, age, neck size, and gender (STOP-Bang), this questionnaire is useful for patients with moderate to severe OSA.

\section{Calgary Sleep Apnea Quality of Life Index}

Calgary Sleep Apnea Quality of Life Index (Calgary SAQLI) was developed by Flemons and Reimer [25] in 1998, and it is a disease-specific quality of life scale for patients with OSA. It evaluates four domains, namely daily functioning (11 questions), social interactions (13 questions), emotional functioning (11 questions), and symptoms of the quality of life (5 questions) that are important to OSA patients. It was designed to be administrated to patients by a trained interviewer. It is rated on a 7-point Likert scale from 1 to 7 points ( $1=$ very severe, $7=$ not at all severe). Calgary SAQLI was translated into a Korean version [26].

\section{CIRCADIAN RHYTHM SLEEP-WAKE DISORDERS}

\section{Morningness-Eveningness Questionnaire}

Morningness-eveningness refers to individual differences in preferred time of activity during daily life, including circadian rhythm, sleep-wake times, and active time zones. Morningness-Eveningness Questionnaire (MEQ) is the most commonly used self-assessment questionnaire to evaluate individual differences in circadian rhythms and was developed by Horne and Osteberg [27] in 1976. The MEQ is a 19 -item questionnaire and the scores range from 16 to 86 . A score of 59 or above indicates morningness and a score below 41 indicates eveningness. MEQ was translated into a Korean version.

\section{Composite Scale of Morningness}

Composite Scale of Morningness (CSM) was developed by Smith et al. [28] by combining MEQ and Diurnal Type Scale for assessing morningness and eveningness. It consists of 13 questions evaluating an individual's preferred sleep onset time, wakeup time, and extent of morning or evening activity. Three items are rated from 1 to 4,10 items are rated from 1 to 5 , and the total score ranges from 13 to 55 . A score over 49 indicates morning type, and a score less than 26 indicates evening type. CSM was translated into a Korean version in 1997 [29].

\section{Sleep Timing Questionnaire}

Sleep Timing Questionnaire (STQ) was developed by Monk et al. [30] to obtain a patient's precise sleep schedule and to obtain the individual's sleep patterns and habits. The questionnaire consists of 18 items; the questions include good night and morning time, number and duration of wake-up during the night, and stability of sleep schedule. STQ has been reported to have a high correlation with actigraphy [30].

\section{RESTLESS LEGS SYNDROME}

\section{International Restless Leg Syndrome Study Group Rating} Scale

International Restless Leg Syndrome Study Group Rating Scale (IRLS) was developed by the International Restless Legs Syndrome Study Group and consists of 10 items to evaluate restless leg syndrome (RLS) symptoms over the previous week [31]. It is designed to evaluate the frequency and severity of RLS, their association with sleep disturbance, daytime functioning, and mood disorder. The degree of severity rated as mild (0 to 10$)$, moderate (11 to 20 ), severe (21 to 30 ), and very severe (31 to 40 ). IRLS was translated into a Korean version in 2010 [32].

\section{Restless Leg Syndrome Quality of Life Questionnaire}

The Restless Leg Syndrome Quality of Life Questionnaire (RLSQoL) was designed to assess the quality of life in the patients with RLS over the previous four weeks. It consists of 18 items including daily activities, social activities, concentration, and sexual activities [33]. RLSQoL was also translated into a Korean version in 2010 [32].

\section{CONCLUSION}

Modern societies are characterized by 24 -hour work operations, trans-meridian travel, and exposure to a variety of electronic devices such as televisions, computers, and mobile phones, all of which affect the quality, quantity, and timing of sleep in human beings [34,35]. Therefore, sleep disorders may gradually increase over time. Early diagnosis and intervention of sleep 
problems or disorders are important. Sleep questionnaires are useful for assessing sleep problems or disorders and for planning treatment, as well as for following up the effect of treatment.

In this paper, we reviewed the characteristics and benefits of some sleep questionnaires that are used in clinical settings. There are some things to be mindful of when using sleep questionnaires. First, sleep questionnaires may not be accurate because almost of them are self-reported and therefore depend on the memory of a particular point in time. Second, some questionnaires have so many items that the rater may evaluate them unintentionally. Third, sleep questionnaires cannot measure sleep stages; they only asses the sleep and awake states [36,37]. Physicians should know about the characteristics and limitations of different sleep questionnaires, and they should combine sleep questionnaires with other evaluation tools when diagnosing patients. Recently, various sleep questionnaires and sleep diaries have been developed into mobile app versions, and consumer wearable devices for easier use [38,39].

In conclusion, if physicians use sleep questionnaires combined with other diagnostic tools, they will be very helpful in identifying sleep problems or disorders of their patients.

\section{Acknowledgments}

None

\section{Conflicts of Interest}

The authors have no potential conflicts of interest to disclose.

\section{Author Contributions}

Conceptualization: Wan Seok Seo. Investigation: Wan Seok Seo, Nayeong Kong, Jinhui Choi. Data curation: Nayeong Kong, Jinhui Choi. Project administration: Wan Seok Seo. Resources: Wan Seok Seo. Supervision: Wan Seok Seo. Writing-original draft: Wan Seok Seo. Writing_-review \& editing: Wan Seok Seo, Nayeong Kong, Jinhui Choi.

\section{ORCID iDs}

Wan Seok Seo (1)

https://orcid.org/0000-0002-5122-5360

Nayeong Kong (1)

https://orcid.org/0000-0002-9095-1701

\section{REFERENCES}

1. Drager LF, McEvoy RD, Barbe F, Lorenzi-Filho G, Redline S; INCOSACT Initiative. Sleep apnea and cardiovascular disease: lessons from recent trials and need for team science. Circulation 2017;136:1840-1850.

2. Tsuno N, Besset A, Ritchie K. Sleep and depression. J Clin Psychiatry 2005;66:1254-1269.

3. Lee EH, Lee SH, Lim YH, Kim W. Investigation and policy development for sleep industry in Gyeonggi-do. Suwon: Gyeonggi Research Institute, 2018.

4. Sateia MJ. International Classification of Sleep Disorders-third edition: highlights and modifications. Chest 2014;146:1387-1394.

5. Abad VC, Guilleminault C. Diagnosis and treatment of sleep disorders: a brief review for clinicians. Dialogues Clin Neurosci 2003;5:371-388.
6. American Sleep Disorders Association. Practice parameters for the indications for polysomnography and related procedures. Sleep 1997;20:406-422.

7. Ibáñez V, Silva J, Cauli O. A survey on sleep questionnaires and diaries. Sleep Med 2018;42:90-96.

8. Rosenthal L, Roehrs TA, Roth T. The Sleep-Wake Activity Inventory: a selfreport measure of daytime sleepiness. Biol Psychiatry 1993;34:810-820.

9. Monk TH, Buysse DJ, Welsh DK, Kennedy KS, Rose LR. A sleep diary and questionnaire study of naturally short sleepers. J Sleep Res 2001;10:173-179.

10. Douglass AB, Bornstein R, Nino-Murcia G, Keenan S, Miles L, Zarcone VP Jr, et al. The Sleep Disorders Questionnaire. I: Creation and multivariate structure of SDQ. Sleep 1994;17:160-167.

11. Morin CM, Vallières A, Ivers H. Dysfunctional Beliefs and Attitudes about Sleep (DBAS): validation of a brief version (DBAS-16). Sleep 2007;30:15471554.

12. Yu ES, Ko YG, Sung GH, Kwon JH. Validation of the Korean version of Dysfunctional Beliefs and Attitudes about Sleep (K-DBAS-16). Korean J Clin Psychol 2009;28:309-320.

13. Buysse DJ, Reynolds CF 3rd, Monk TH, Berman SR, Kupfer DJ. The Pittsburgh Sleep Quality Index: a new instrument for psychiatric practice and research. Psychiatry Res 1989;28:193-213.

14. Sohn SI, Kim DH, Lee MY, Cho YW. The reliability and validity of the Korean version of the Pittsburgh Sleep Quality Index. Sleep Breath 2012;16:803-812.

15. Morin CM, Belleville G, Bélanger L, Ivers H. The Insomnia Severity Index: psychometric indicators to detect insomnia cases and evaluate treatment response. Sleep 2011;34:601-608.

16. Cho YW, Song ML, Morin CM. Validation of a Korean version of the Insomnia Severity Index. J Clin Neurol 2014;10:210-215.

17. Johns MW. A new method for measuring daytime sleepiness: the Epworth Sleepiness Scale. Sleep 1991;14:540-545.

18. Cho YW, Lee JH, Son HK, Lee SH, Shin C, Johns MW. The reliability and validity of the Korean version of the Epworth Sleepiness Scale. Sleep Breath 2011;15:377-384.

19. Jung SY, Cho CH, Rhee MK, Kim L, Lee HJ. Development of a modified Korean version of the Epworth Sleepiness Scale reflecting Korean sociocultural lifestyle. Psychiatry Investig 2018;15:687-694.

20. Hoddes E, Zarcone V, Smythe H, Phillips R, Dement WC. Quantification of sleepiness: a new approach. Psychophysiology 1973;10:431-436.

21. Netzer NC, Stoohs RA, Netzer CM, Clark K, Strohl KP. Using the Berlin Questionnaire to identify patients at risk for the sleep apnea syndrome. Ann Intern Med 1999;131:485-491.

22. Tan A, Yin JD, Tan LW, van Dam RM, Cheung YY, Lee CH. Using the Berlin Questionnaire to predict obstructive sleep apnea in the general population. J Clin Sleep Med 2017;13:427-432.

23. Kang K, Park KS, Kim JE, Kim SW, Kim YT, Kim JS, et al. Usefulness of the Berlin Questionnaire to identify patients at high risk for obstructive sleep apnea: a population-based door-to-door study. Sleep Breath 2013;17:803810.

24. Chung F, Yegneswaran B, Liao P, Chung SA, Vairavanathan S, Islam S, et al. STOP questionnaire: a tool to screen patients for obstructive sleep apnea. Anesthesiology 2008;108:812-821.

25. Flemons WW, Reimer MA. Development of a disease-specific health-related quality of life questionnaire for sleep apnea. Am J Respir Crit Care Med 1998; 158:494-503.

26. Jang HU, Park KS, Cheon SM, Lee HW, Kim SW, Lee SH, et al. Development of the Korean version of the Sleep Apnea Quality of Life Index. Clin Exp Otorhinolaryngol 2014;7:24-29.

27. Horne JA, Ostberg O. A self-assessment questionnaire to determine morningness-eveningness in human circadian rhythms. Int J Chronobiol 1976;4:97-110.

28. Smith CS, Reilly C, Midkiff K. Evaluation of three circadian rhythm questionnaires with suggestions for an improved measure of morningness. J Appl Psychol 1989;74:728-738.

29. Yoon JS, Shin SM, Kook SH, Lee HY. A preliminary study on the Korean translation of Composite Scale (KtCS) to measure morningness-eveningness. J Korean Neuropsychiatr Assoc 1997;36:122-134.

30. Monk TH, Buysse DJ, Kennedy KS, Pods JM, DeGrazia JM, Miewald JM. Measuring sleep habits without using a diary: the Sleep Timing Questionnaire. Sleep 2003;26:208-212.

31. Walters AS. Toward a better definition of the restless legs syndrome. The In- 
ternational Restless Legs Syndrome Study Group. Mov Disord 1995;10:634642.

32. Yang JG, Kim DH, Lee JH, Park KH, Jung KY, Shin WC, et al. The reliability and validity of the Korean versions of the International Restless Legs Scale and the Restless Legs Syndrome Quality of Life Questionnaire. J Korean Neurol Assoc 2010;28:263-269.

33. Abetz L, Vallow SM, Kirsch J, Allen RP, Washburn T, Earley CJ. Validation of the Restless Legs Syndrome Quality of Life questionnaire. Value Health 2005;8:157-167.

34. Shochat T. Impact of lifestyle and technology developments on sleep. Nat Sci Sleep 2012;4:19-31.

35. Lee HJ. Chronobiology, the future of medicine. Chronobiol Med 2019;1:1-2.

36. Lim YH, Choi J, Kim KR, Shin J, Hwang KG, Ryu S, et al. Sex-specific characteristics of anthropometry in patients with obstructive sleep apnea: neck circumference and waist-hip ratio. Ann Otol Rhinol Laryngol 2014;123: 517-523.

37. Nishiyama T, Mizuno T, Kojima M, Suzuki S, Kitajima T, Ando KB, et al. Criterion validity of the Pittsburgh Sleep Quality Index and Epworth Sleepiness Scale for the diagnosis of sleep disorders. Sleep Med 2014;15:422-429.

38. Lee XK, Chee NIYN, Ong JL, Teo TB, van Rijn E, Lo JC, et al. Validation of a consumer sleep wearable device with actigraphy and polysomnography in adolescents across sleep opportunity manipulations. J Clin Sleep Med 2019;15:1337-1346.

39. Hekler EB, Buman MP, Grieco L, Rosenberger M, Winter SJ, Haskell W, et al. Validation of physical activity tracking via android smartphones compared to ActiGraph accelerometer: laboratory-based and free-living validation studies. JMIR Mhealth Uhealth 2015;3:e36. 